sis from Alberta, Canada. Blood. 2020:136(Suppl 1):26-27.

2. Orellana-Noia VM, Reed DR, Sen JM, et al. CNS prophylaxis during front-line therapy in aggressive non-Hodgkin lymphomas: realworld outcomes and practice atterns from 19 US academic nstitutions. Blood. 2020;136(Suppl 1):27-28.

3. Eyre TA, Djebbari F, Kirkwood AA, Collins GP. Efficacy of central nervous system prophylaxis with stand-alone intrathecal chemotherapy in diffuse large B-cell lymphoma patients treated with anthracycline-based chemotherapy in the rituximab era: a systematic review. Haematologica. 2020;105(7):1914-1924.

4. Eyre TA, Kirkwood AA, Wolf J, et al. Stand-alone intrathecal central nervous system (CNS) prophylaxis provide unclear benefit in reducing CNS relapse risk in elderly DLBCL patients treated with R$\mathrm{CHOP}$ and is associated increased infection-related toxicity. Br J Haematol. 2019;187(2):185-194.

5. Cheah CY, Herbert KE, O'Rourke K, et al. A multicentre retrospective comparison of central nervous system prophylaxis strategies among patients with high-risk diffuse large B-cell lymphoma. Br J Cancer. 2014:111(6):1072-1079.

6. Schmitz R, Wright GW, Huang DW, et al. Genetics and pathogenesis of diffuse large B-cell lymphoma. N Engl J Med. 2018;378(15):13961407.

7. Chapuy B, Stewart C, Dunford AJ, et al. Molecular subtypes of diffuse large B cell lymphoma are associated with distinct pathogenic mechanisms and outcomes. Nat Med. 2018;24(5):679-690.

8. Wright GW, Huang DW, Phelan JD, et al. A probabilistic classification tool for genetic subtypes of diffuse large $B$ cell lymphoma with therapeutic implications. Cancer Cell. 2020;37(4):551-568.e14.

9. Schmitz N, Zeynalova S, Nickelsen M, et al. CNS International Prognostic Index: a risk model for CNS relapse in patients with diffuse large B-cell lymphoma treated with R-CHOP. J Clin Oncol. 2016;34(26):3150-3156.

10. Klanova M, Sehn LH, Bence-Bruckler I, et al. Integration of cell of origin into the clinical CNS International Prognostic Index improves CNS relapse prediction in DLBCL. Blood. 2019;133(9):919-926.

11. Arvanitis CD, Ferraro GB, Jain RK. The blood-brain barrier and blood-tumour barrier in brain tumours and metastases. Nat Rev Cancer. 2020;20(1):26-41.

12. Abramson JS, Hellmann M, Barnes JA, et al. Intravenous methotrexate as central nervous system (CNS) prophylaxis is associated with a low risk of CNS recurrence in high-risk patients with diffuse large Bcell lymphoma. Cancer. 2010;116(18):4283-4290.

13. McKay P, Wilson MR, Chaganti S, Smith J, Fox CP, Cwynarski K. British Society of Haematology. The prevention of central nervous system relapse in diffuse large B-cell lymphoma: a British Society for Haematology good practice paper. Br J Haematol. 2020;190(5):708714.

14. Ambady P, Holdhoff M, Bonekamp D, Wong F, Grossman SA. Late relapses in primary CNS lymphoma after complete remissions with high-dose methotrexate monotherapy. CNS Oncol. 2015;4(6):393398.

15. Swerdlow SH, Campo E, Pileri SA, et al. The 2016 revision of the World Health Organization classification of lymphoid neoplasms. Blood. 2016;127(20):2375-2390.

16. Ollila TA, Kurt H, Waroich J, et al. Genomic subtypes may predict the risk of central nervous system recurrence in diffuse large B-cell lymphoma. Blood. 2021;137(8):1120-1124

17. Twa DDW, Lee DG, Tan KL, et al. Genomic predictors of central nervous system relapse in primary testicular diffuse large B-cell lymphoma (DLBCL). Blood. 2021;137(9):1256-1259.

18. Lionakis MS, Dunleavy K, Roschewski M, et al. Inhibition of B cell receptor signaling by ibrutinib in primary CNS lymphoma. Cancer Cell. 2017;31(6):833-843.e5.

19. Grommes C, Pastore A, Palaskas N, et al. Ibrutinib unmasks critical role of Bruton tyrosine kinase in primary CNS lymphoma. Cancer Discov. 2017;7(9):1018-1029

20. Younes A, Sehn LH, Johnson P, et al. Randomized phase III trial of ibrutinib and rituximab plus cyclophosphamide, doxorubicin, vincristine, and prednisone in non-germinal center B-cell diffuse large Bcell lymphoma. J Clin Oncol. 2019;37(15):1285-1295.

21. Ghesquieres $\mathrm{H}$, Chevrier $M$, Laadhari $M$, et al. Lenalidomide in combination with intravenous rituximab (REVRI) in relapsed/refractory primary CNS lymphoma or primary intraocular lymphoma: a multicenter prospective 'proof of concept' phase II study of the French Oculo-Cerebral Lymphoma (LOC) Network and the Lymphoma Study Association (LYSA). Ann Oncol. 2019;30(4):621-628.

22. Nowakowski GS, Hong F, Scott DW, et al. Addition of lenalidomide to R-CHOP improves outcomes in newly diagnosed diffuse large Bcell lymphoma in a randomized phase II US Intergroup Study ECOG-ACRIN E1412. J Clin Oncol. 2021;39(12):1329-1338.

\title{
All in the family: back-to-back kinase inhibitors for the treatment of chronic lymphocytic leukemia
}

\author{
Meghan C. Thompson, Lindsey E. Roeker and Anthony R. Mato \\ Leukemia Service, Memorial Sloan Kettering Cancer Center, New York, NY, USA \\ E-mail: ANTHONY R. MATO - matoa@mskcc.org
}

doi:10.3324/haematol.2021.278535

I this issue of Haematologica, Rogers et al. address a key sequencing question in the management of chronic lymphocytic leukemia (CLL) by reporting the results of the largest prospective clinical trial evaluating acalabrutinib for the treatment of CLL following intolerance to ibrutinib. ${ }^{1}$ While the Bruton tyrosine kinase (BTK) inhibitor ibrutinib has led to a paradigmatic shift in the treatment of CLL away from chemoimmunotherapy, high rates of ibrutinib discontinuation remain a major problem.

Real-world evidence and long-term follow-up from clinical trials of ibrutinib have established that drug intolerance due to toxicity, rather than progressive CLL, is the most common reason for discontinuation of ibrutinib treatment. ${ }^{2-4}$ Real-world data from 616 CLL patients treated with ibrutinib in clinical practice documented that $41 \%$ of patients discontinued ibrutinib (median follow- up 17 months), and more than half of all discontinuations were due to toxicity. ${ }^{2}$ Real-world evidence from the UK documents high rates of ibrutinib discontinuation due to reasons other than disease progression $(17.5 \%){ }^{3}$ Furthermore, similar patterns have emerged with longer follow-up data from clinical trials, with more patients discontinuing ibrutinib due to toxicity than because of CLL progression. At 5 years of follow-up of the RESONATE-2 trial of ibrutinib for initial treatment of CLL, $41 \%$ of patients had discontinued ibrutinib therapy, with a $21 \%$ discontinuation rate due to adverse events including atrial fibrillation. ${ }^{4}$ Furthermore, in a pooled analysis of CLL patients treated with ibrutinib on three randomized phase III studies, $11 \%$ of patients permanently discontinued ibrutinib due to adverse events and $13 \%$ of patients required dose reductions due to adverse events, highlighting the significant impact of adverse events while on 


\section{TREATMENT OF CLL AFTER INTOLERANCE TO IBRUTINIB}

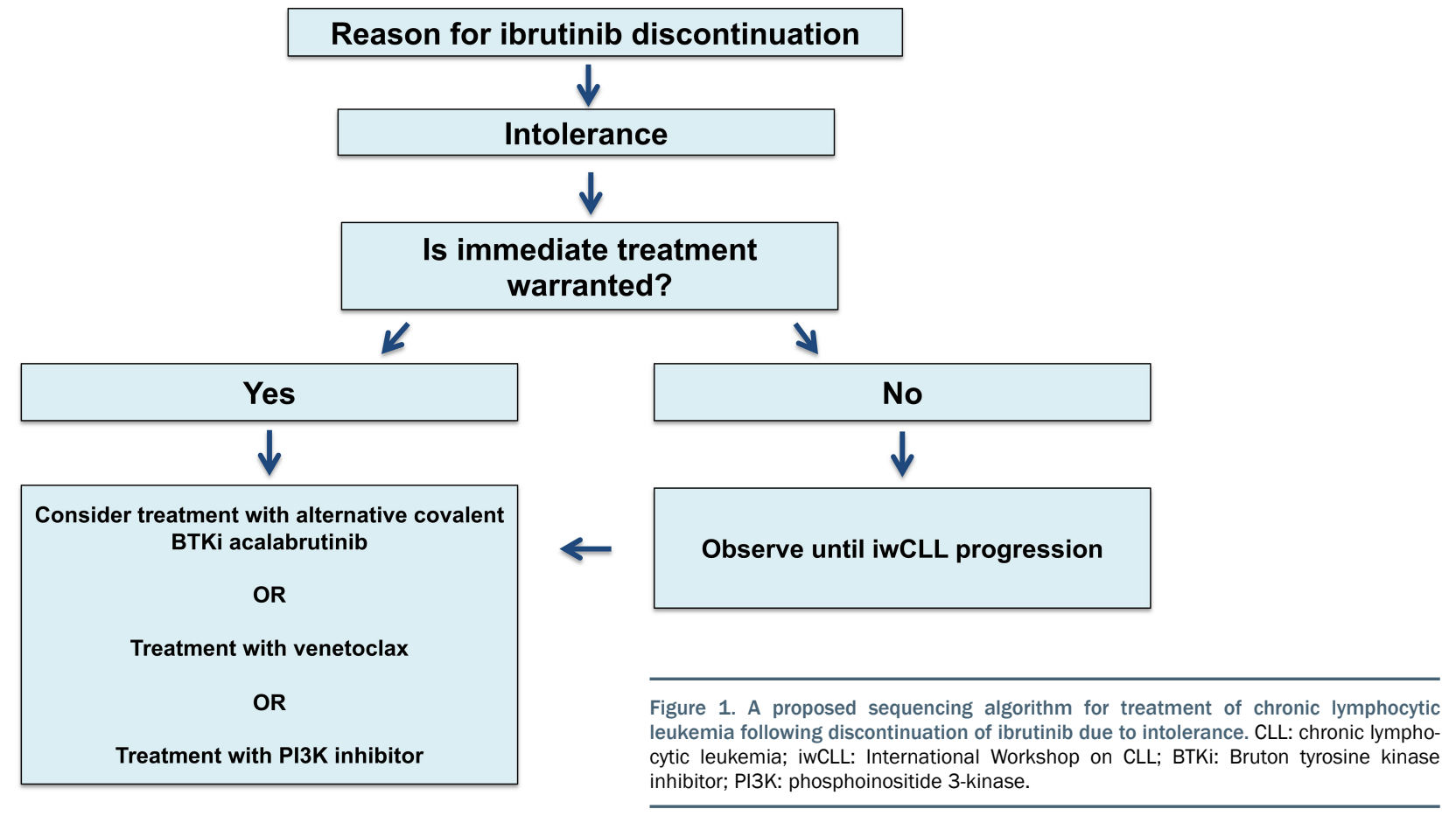

treatment with ibrutinib. ${ }^{5}$ These studies clearly established that intolerance to ibrutinib is a common scenario encountered in clinical practice, which may limit the clinical benefit of this drug that has been largely studied as a continuous therapy.

Given the clinical efficacy of BTK inhibition in CLL, for patients who discontinue a BTK inhiibitor due to intolerance, an important question is whether treatment with an alternative kinase inhibitor is an acceptable treatment option. This is particularly relevant given the development of more selective BTK inhibitors with fewer off-target effects. Newer BTK inhibitors include approved therapies such as acalabrutinib, as well as emerging covalent and non-covalent BTK inhibitors in clinical development (zanubrutinib, LOXO-305, ARQ-351).

Previously, Awan et al. addressed this key question by conducting a small cohort study of acalabrutinib treatment for patients who discontinued ibrutinib due to intolerance (defined by the investigator's discretion). ${ }^{6}$ In this study of 33 patients, the efficacy of acalabrutinib following ibrutinib was high (overall response rate $76 \%$ ) with only $9 \%$ of patients discontinuing acalabrutinib due to an adverse event. ${ }^{6}$ However, this study examined only a small number of patients and lacked an objective definition of ibrutinib intolerance.

The study by Rogers et al. is the first prospectively designed study to answer this important sequencing question. ${ }^{1}$ Intolerance was defined as discontinuation of ibrutinib due to either persistent/recurrent grade 2 adverse events despite dose modification or interruption or persistent grade $3 / 4$ adverse events. Sixty patients with relapsed and/or refractory CLL were treated with acalabrutinib (median number of prior therapies 2) with a prior median duration of ibrutinib therapy of 5.7 months. Overall, the approach was well-tolerated, with the most common adverse events being diarrhea (53\%), headache (42\%) and contusion (40\%). Only $40 \%$ of patients had ibrutinib-related intolerance adverse events, and $67 \%$ of events were lower grade with acalabrutinib than with ibrutinib; only one adverse event (increased levels of liver enzymes) occurred at a higher grade with acalabrutinib treatment than with ibrutinib treatment. Notably, more patients discontinued acalabrutinib because of CLL progression $(23 \%)$ than because of adverse events $(17 \%)$. Acalabrutinib following discontinuation of ibrutinib for intolerance was efficacious, with an overall response rate of $73 \%$ and a 24 -month estimated progression-free survival of $72 \%$ (median follow-up, 35 months). It should be noted that the majority (94\%) of patients with available pre-treatment sequencing data did not have BTK or PLCG2 mutations prior to initiating treatment with acalabrutinib. ${ }^{1}$

In addition to the work presented by Rogers et al., two additional recent studies have also shown that treatment of CLL with an alternative kinase inhibitor following ibrutinib intolerance is safe and efficacious. ${ }^{7,8}$ A phase II study examined the phosphoinositide 3-kinase (PI3K) inhibitor umbralisib in 51 patients with relapsed/refractory CLL who were intolerant to prior BTK inhibition $(n=44)$ or PI3K inhibition $(n=7)$ and showed a median progression-free survival of 23.5 months, with the majority $(58 \%)$ of patients remaining on umbralisib for longer than on their prior kinase inhibitor therapy. Additionally, LOXO-305 (pirtobrutinib), a novel, highly selective, noncovalent BTK inhibitor showed a favorable safety profile in 170 patients with CLL/small lymphocytic leukemia, of 
whom $86 \%$ had received prior treatment with a BTK inhibitor, with $33 \%$ discontinuing the prior BTK inhibitor due to reasons other than progressive CLL. ${ }^{8}$ Furthermore, LOXO-305 had promising efficacy in this heavily pretreated population with an overall response rate of $62 \%$ in 121 efficacy-evaluable patients with CLL/small lymphocytic leukemia who had previously been treated with a BTK inhibitor. ${ }^{8}$

Taken together, these studies challenge the traditional sequencing paradigm of switching drug classes in the setting of CLL therapy discontinuation for intolerance. In Figure 1, we propose a sequencing algorithm incorporating the new data from Rogers et al. While venetoclax is an acceptable option in the setting of intolerance to BTK inhiitors, ${ }^{9}$ CLL remains an incurable, chronic disease and there is a strong scientific rationale for maximizing clinical benefit from each drug class prior to exposing patients to the selective pressures of another therapeutic class. In the case of the common problem of intolerance to ibrutinib it is best to keep the solution "all in the (BTK inhibitor) family."

\section{Disclosures}

The authors have no disclosures to make regarding this editorial. With regard to work outside this publication, MCT has received honoraria from MJH Life Sciences, VJHemOnc, and Curio Science. LER has received research funding from the American Society of Hematology and Pfizer; has minority ownership interest in Abbott Laboratories; provides consultancy services for AbbVie, AstraZeneca, Pharmacyclics, the Vaniam group and, uncompensated, for Verastem. ARM has received grants and personal fees from and is a data safety monitoring board member for TG Therapeutics; has received grants and personal fees from Loxo Oncology (a wholly owned subsidiary of Eli Lilly), Genentech, AbbVie, AstraZeneca, Adaptive,
Pharmacyclics, and Curio Sciences; has received grants from Sunesis, Regeneron, Pfizer, Aprea, Aptose, and DTRM; has received non-financial support from the NCCN, CLL society, and Lymphoma Research Foundation; and has received grants from and sat on a steering committee for Verastem.

\section{Contributions}

MCT and ARM drafted the manuscript. MCT, LER and $A R M$ provided feedback and edited the manuscript.

\section{References}

1. Rogers KA, Thompson PA, Allan JN, et al. Phase II study of acalabrutinib in ibrutinib-intolerant patients with relapsed/refractory chronic lymphocytic leukemia. Haematologica. 2021;106(9):2364-2373.

2. Mato AR, Nabhan C, Thompson MC, et al. Toxicities and outcomes of 616 ibrutinib-treated patients in the United States: a real-world analysis. Haematologica. 2018;103(5):874-879.

3. UK CLL Forum. Ibrutinib for relapsed/refractory chronic lymphocytic leukemia: a UK and Ireland analysis of outcomes in 315 patients. Haematologica. 2016;101(12):1563-1572

4. Burger JA, Barr PM, Robak T, et al. Long-term efficacy and safety of first-line ibrutinib treatment for patients with CLL/SLL: 5 years of follow-up from the phase 3 RESONATE-2 study. Leukemia. 2020;34(3):787-798.

5. Coutre SE, Byrd JC, Hillmen P, et al. Long-term safety of single-agent ibrutinib in patients with chronic lymphocytic leukemia in 3 pivotal studies. Blood Adv. 2019;3(12):1799-1807.

6. Awan FT, Schuh A, Brown JR, et al. Acalabrutinib monotherapy in patients with chronic lymphocytic leukemia who are intolerant to ibrutinib. Blood Adv. 2019;3(9):1553-1562.

7. Mato AR, Ghosh N, Schuster SJ, et al. Phase 2 study of the safety and efficacy of umbralisib in patients with CLL who are intolerant to

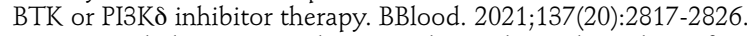

8. Mato AR, Shah NN, Jurczak W. Pirtobrutinib in relapsed or refractory B-cell malignancies (BRUIN): a phase $1 / 2$ study. Lancet. 2021;397(10277):892-901.

9. Jones JA, Mato AR, Wierda WG, et al. Venetoclax for chronic lymphocytic leukaemia progressing after ibrutinib: an interim analysis of a multicentre, open-label, phase 2 trial. Lancet Oncol. 2018;19(1):65-75.

\section{Do we need more genome wide association studies?}

\section{Stephan Menzel}

Red Cell Research Unit, King's College London, London, UK E-mail: STEPHAN MENZEL - stephan.menzel@kcl.ac.uk

doi:10.3324/haematol.2021.278642

M uch of the individual biological traits we have, of what we look like, of our physical and mental abilities, of our risk to suffer from the noncommunicable diseases that will ultimately end our lives, is encoded in the genetic 'background', consisting of millions of single-nucleotide polymorphisms (SNP) and other common sequence variants that each have minute functional effects on regulatory sequences within our genome.

Genome-wide association studies (GWAS) are the tool of choice to make the connection between commonvariant genotype data, collected either through genome sequencing or with genotyping arrays ('chips'), and human phenotype. In its simplest form, GWAS compare the frequency for each of of thousands or millions of common genetic variants between groups of patients and controls, thus identifying genetic risk factors for the diseases studied this way.

There are limits to what the traditional GWAS approach can achieve. Suffocating type-I error rates arising from the analysis of millions of genetic variants make it necessary to assemble very large groups of patients and controls, but even then, only the strongest genetic risk factors can be identified with meaningful certainty. Even so, finding this initial set of genetic factors has significantly enhanced our understanding of pathways leading to common disease or shaping healthrelevant physiological traits. With the majority of disease risk factors still hidden, however, it is presently impossible to assemble enough genetic information to 